\title{
AN INTEGRATED FRAMEWORK OF LEADERSHIP FOR HEALTHCARE ORGANIZATIONS TO NAVIGATE THROUGH COVID-19 CRISIS
}

\author{
Mukul Kumar Jha \\ Tata Institute of Social Sciences, Mumbai, India
}

Correspondence:mukuljha37@gmail.com

\begin{abstract}
COVID-19 crisis has strained healthcare systems immensely creating a multi-front challenge to overcome. Healthcare leaders face stressful situations like long arduous hours of work, isolation from their loved ones, immense mental health issues along with fighting false narratives and campaigns by social media. Hence, there is a dire need for leaders to embrace this uncertainty and evolve by adopting a strategic shift in their mindset.
\end{abstract}

To propose an effective functional leadership model of practice during crisis, author has undertaken a qualitative approach to understand the various literature published on crisis management, reviewed the literature on healthcare leadership, contextualized the papers about unique challenges posed by a crisis like COVID-19, and utilize the learnings to design an integrated framework for healthcare organizations to be applied during a crisis.

Author presents a systems-based view of leadership challenges in healthcare organizations during a crisis and proposes a unique framework of 3A- Acknowledge, Activate, and Agility which could serve a comprehensive tool of strategic leadership for healthcare leaders to adopt during a crisis. When healthcare organizations adopt the princi ples embedded within $3 \mathrm{~A}$ model elements, it would help them realize better patient outcomes, develop compassionate organizational culture, and enhance professional satisfaction within their teams.

\section{KEYWORDS}

COVID-19, Crisis leadership, psychological safety, acknowledge, patient-centric care, agility, activate.

\section{INTRODUCTION}

The COVID-19 crisis has severely tested healthcare leaders across the globe on multiple fronts be it saving lives, sustaining essential medical supplies, caring for their own staff, engaging with customer, or enabling smooth business processes to sustain efficient operations. Healthcare leaders face stressful situations like long arduous hours of work, isolation from their loved ones, immense mental health issues, fighting false narratives and malicious campaigns by social media.[1] Such convoluted trajectory of pandemic has given rise to a spike in the sense of loss of control and has heightened emotional vulnerability due to the high amount of uncertainty. While pandemic has still 
not subsided, it has exposed deep structural inefficiencies of health systems and has created an overshoot in need for leaders who can help us navigate through such tortuous times.

But, why is leadership in a crisis so different from normal times? Crisis like COVID-19 pose herculean challenges with crisis experts like Mitroff, Boin etc. highlighting the unique challenges of chaos, ambiguity, and stress in crisis. [2,3] Yuk and Zaleznik have emphasized the importance of leadership during any crisis.[4] Bernard Bass has explicitly studied crisis leadership in business context and recommended that a crisis demands flexible and adaptive leadership style.[5] Such an approach includes a mixture of being assertive, directive as well as trusting the team members through decentralized approach. Healthcare organizations often tend to be hindered by the authoritative and expert based leaders who hold onto authority and fail to decentralize the various sub-systems of organization. Arjen Boin and Paul t' Hart have addressed the issues associated with such a mindset and recommends that a crisis requires multi-agency coordination and collaboration among agencies which may not have worked together before and leader has to channel such coordination.[3] Demiroz et al.' identified unique traits of a leader to include "cooperation, adaptability, flexibility and communication" in crisis times.[6] R. Heifetz, in his famous book "Leadership without easy answers", has tabulated the "technical" and "'adaptive" problems that a physician needs to solve to navigate in times when it is tough to make decisions.[7] Despite the presence of a vast academic literature on leadership during crisis, they were mostly limited to emphasizing on just few limited parameters thereby lacking the integrative and comprehensive nature of leadership practices. This article attempts to provide novel integrated framework of healthcare leadership which shall serve as handy and easy to use guide for managing complexities during a crisis in healthcare settings.

It is important to look at leadership challenges as distinct from the case of routine emergency situations in healthcare settings. [8] Shingler-Nace had identified 5 ingredients for an effective leadership during crisiscalmness, communication, collaboration, coordination and providing support.[9] Physicians and administrators need to evolve these qualities within the new context of pandemic and ensure that they abide by their core duties with utmost integrity and dedication. Before proceeding with the model for crisis leadership, let us look at how
COVID-19 highlighted the deficiencies in critical leadership skills. COVID-19 brought massive overload on the existing health infrastructure in terms of the scarcity of beds, ICUs, ventilators, oxygen cylinders and even basic medicines for day-to-day use. Healthcare workers were stretched to work for double shifts in many cases thereby straining their physical endurance and subjecting them to extreme physical stress. Emotional health of such workers was equally jeopardized by being isolated from their families and seeing exhausting number of deaths every day.[1] Managing emotionally volatile relatives of patients was an additional draining aspect for healthcare leaders.

A crisis of the scale and complexity of COVID-19 demands a transformational shift in the mindset of leaders. An effective healthcare leader in such crisis situations must encompass few major dimensions to his leadership arsenal:

\section{SENSE OF SELF}

i) Patience and observation: Leaders need to wait, refrain from impulsive action under stress, observe, have conversations to identify meaningful actionable themes to emerge.

ii) Resilience: A mindset that enables adoption of resilience and agility is critical to adapt quickly to new demands of pandemic. It also includes emotional resilience which provides a deeper "sense of purpose" to these healthcare leaders and stay motivated to persevere amidst recurring failures.

iii) Shift from expert to an enabler: Avoid "I know it all" approach and trust their teams for their ability to execute decisions. They will have to place trust in the on-the ground stakeholders and influencers who can act on the insights derived from patients, nurses, medical suppliers, and govt.

\section{HONESTY LEADING TO INTEGRITY:}

Healthcare organizations need to be honest in the cost they charge from patients for treatments recognizing that a vast majority of the patients' hail from poor socioeconomic strata of society. Cost of test, hospitalization and ICU beds should be rationally charged and patients should be informed about the expenses and billings upfront to help them prepare better. Deeper commitment to honesty comforts patients that healthcare leaders are trustworthy and despite the common calamity, they are there for them through their thick and thin. 


\section{PSYCHOLOGICAL SAFETY:}

Decentralized approach to leadership demands that leaders make every person feel valued and respected for their duties and welcome them to take part in active decision making. Leaders are under tremendous pressure from govt and international organizations and often pass on this stress to their team members failing to understand the concerns going on within their team internally. Team members, despite undergoing extreme anxiety, hesitate to freely expresses their emotions and as a result end up being more miserable. It is the job of leader that they let their team members not subjugate under pressure and foster a climate of psychological safety by enabling every member to feel included within their decisions.

Crisis demands that leaders recognize the crisis, acknowledge its severity, and plan a strategy for its shortterm and long-term impact. It needs leaders, ideally with medical knowledge and experience in administration, to lead from the front. By encouraging shared and collaborative learning from all experienced medical professionals in teams, a leader who empowers his teammates shall be the one who synergizes a result driven healthcare organization.

An essential point to consider here is that many leaders may dive into "dilemma thinking it is better not to act at all and observe till the pandemic resides". This could be as disastrous as doing nothing and can put their teams under far more stress and anxiety. As per one of the landmark studies conducted in social psychology titled, "The Challenges of the Disengaged Mind," it was found that participants preferred to give themselves electric shocks instead of being left in isolation with their thoughts.[10] Leaders need to learn that when followers see them taking even minor actions like communicating frequently and asking for feedback, it can be more helpful and psychologically uplifting than just sitting idle.

After we have seen the critical dimensions of healthcare leader's principles, I propose below a model of 3AAcknowledge, Activate and Agility which could serve as easy-to-use framework to categorize the various decisions leaders take and enhance their efficacy.

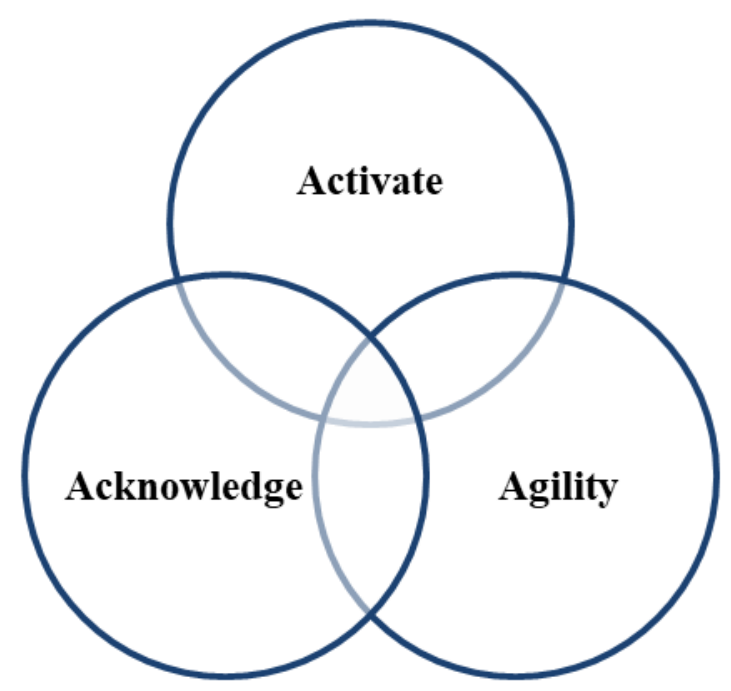

\section{ACKNOWLEDGE}

Resilient leaders zero in on the most pressing issues, amidst a flurry of urgent issues, and establish priority areas. Leaders have to embrace the uncertainty and publicly acknowledge that they may not have all the answers. Such a public acknowledgement enables employees to see the human side of their leaders and motivates others to step up in search for the right answer.
SUPPORT TALENT AND STRATEGY:

Organizations must see that all their "talents" align themselves with the purpose of the organisation by acknowledging the severity of crisis and step up to cater to the additional workload. When companies are centered on an authentic purpose, employees feel that their work has meaning. Research shows that employees who feel a greater sense of connection are far more likely to ride out volatility and be there to help companies recover and grow. 


\section{ENGAGE WITH YOUR BUSINESS ECOSYSTEM:}

Employees at each level of the organization have to understand the concerns of the other departments and evolve their practices with the evolving business ecosystem. Acknowledging the integrated impact of one division's activities over the other can help break operational siloes and enable seamless integration for maximum effectiveness during a crisis.

\section{STAY ENGAGED WITH CUSTOMERS:}

Healthcare organizations need to focus on determining the actions to preserve their customers by developing a responsive patient support system. They should simultaneously look for new avenues for catering to patient's shifting needs by adopting innovative business models based on tele-health, home care etc.

\section{ACTIVATE}

Leaders will have to rapidly deploy a crisis command centre which would serve as the nodal centre to maintain consistency of information and efficiency in communicating decisions of leaders. A detailed risk analysis categorizing various actions related to process, people and structure which are essential to cater to the evolving needs of crisis must be initiated proactively.

\section{SAFETY OF EMPLOYEES AS FIRST LINE OF DEFENCE:}

Organizations must ensure that they lay down set of guidelines and precautionary measures to be followed strictly by the staff, compensate staff accordingly, plan for their safe transport and ensure safe living conditions for them. The employees need to be provided proper meals on time, allocated duties with adequate assurance that their health shall be the core priority of healthcare firm. Simultaneously, the employees shall also have to be trained properly to follow all precautionary measures and to take ownership of their own safety as well as that of the others.

\section{STRENGTHEN DIGITAL CAPABILITIES \& OPPORTUNITIES:}

Digital infrastructure helps develop integrated userexperience, monitor condition of patients remotely help reduce the burden on healthcare infrastructure. It is vital to develop database of patient's care and enable more data-centric decision making.

\section{SHORE UP THE SUPPLY CHAIN AND INVENTORY MANAGEMENT:}

Healthcare leaders will have to be empathetic to their medical suppliers, dealers, partners and assist them in their transition to build a sense of trust and long-term loyalty. Crisis demands that health organizations collaborate and deepen the existing relationship through sound operations management practices.

\section{AGILITY}

Since expediency is essential, leaders need to adapt and change constantly to evolve to cater to the new demands of the crisis. In one analysis firms from 1955 to 2014, it was found that around $88 \%$ of companies which were in the Fortune 500 list in 1955 were gone by the time 2014 arrived.[ [1 1 ] It shows the importance of evolving with time and adopt change management principles within healthcare. Healthcare leaders often go into inertia with their "reputed past" and fail to recognize that "what brought them here, won't take them there". Organizations will have to adopt newer emerging business models like more day care, home care facilities, tele health services. Such models are essential for organizations to operate on a sustainable profit margin, cut cost while also delivering due patient care services. Agility does not refer to just organizational agility, but also emotional agility that leaders need to embrace. Leaders must become more collaborative within their teams, communicate frequently, be compassionate, learn from others and be willing to change their outlook towards healthcare reforms.

\section{CONCLUSION AND WAY FORWARD}

Healthcare leaders will have to imagine themselves as captain of a ship which has lost its way in the storm and does not have a compass to find out the right direction. It is only through utmost grit, deep conviction, and collaborative leadership that the captain can help crew sail through the storm. While leaders may still become skeptical of their own decisions, they must hold themselves accountable, take complete ownership and motivate their employees by practicing humility, emotional resilience, and soft-leadership principles to enable their teams develop a culture of "we all are in it together". No one knows how the pandemic will bring transformational changes in our lives but if organizations invest in developing values and behaviors that can support people and communities towards easing out their pain, it would certainly help us emerge stronger with a reformed relationship and rejuvenated "patient-care" model. 


\section{References:}

1. Shreffler J, Huecker M, Petrey J. The Impact of COVID19 on Healthcare Worker Wellness: A Scoping Review. Western Journal of Emergency Medicine. 2020;21 (5).

2. Mitroff I. Crisis leadership. Hoboken, N.J: Wiley; 2004.

3. Boin A. The politics of crisis management. Cambridge: Cambridge University Press; 2017.

4. Zaleznik. "Managers and Leaders: Are They Different,". Harvard Business Review [Internet]. 2004 [cited 28 April 2021]; Available from:

https://hbr.org/2004/01/managers-and-leaders-arethey-different

5. Bass B, Avolio B, Jung D, Berson Y. Predicting unit performance by assessing transformational and transactional leadership. Journal of Applied Psychology. 2003;88(2):207-218.

6. DemirozF, Kapucu N. The Role of Leadership in Managing Emergencies and Disasters. European Journal of Economic and Political Studies [Internet]. 2012 [cited 28 April 2021];5(1). Available from: https://arastirmax.com/tr/system/files/dergiler/25821/ makaleler/5/1/arastirmax-role-leadership-managingemergencies-and-disasters.pdf

7. Heifetz R. Leadership Without Easy Answers. Cambridge: Harvard University Press; 2009.

8. H.F. A, Thayaparan M, Kulatunga U. Understanding the terminologies: Disaster, Crisis and Emergency. Proceedings of 32nd Annual ARCOM Conference [Internet]. Manchester: Association of researchers in construction management; 2016 [cited 28 April 2021]. Available from: https://www.arcom.ac.uk/docs/proceedings/9ac79958d9024495cd81e13909ed0 8cb.pdf

9. Shingler-Nace A. COVID-19: When Leadership Calls_Letter to Editor. Nurse Leader. 2020;18(3):202-203.

10. Wilson T, Reinhard D, Westgate E, GilbertD, Ellerbeck $\mathrm{N}$, Hahn $\mathrm{C}$ et al. Just think: The challenges of the disengaged mind. Science. 2014;345(6192):75-77.

11. Perry M. Fortune 500 firms in 1955 vs. $2014 ; 88 \%$ are gone, and we're all better off because of that dynamic 'creative destruction' [Internet]. aei.org. 2014 [cited 28 April 2021]. Available from: https://www.aei.org/carpe-diem/fortune-500-firms-in1955-vs-2014-89-are-gone-and-were-all-better-offbecause-of-that-dynamic-creative-destruction/ 